\title{
Active-passive calibration of optical tweezers in viscoelastic media
}

Fischer, Mario; Richardson, Andrew C; S Reihani, S Nader; Oddershede, Lene; Berg-Sørensen, Kirstine

Published in:

Review of Scientific Instruments

Link to article, DOI:

$10.1063 / 1.3280222$

Publication date:

2010

Document Version

Publisher's PDF, also known as Version of record

Link back to DTU Orbit

Citation (APA):

Fischer, M., Richardson, A. C., S Reihani, S. N., Oddershede, L., \& Berg-Sørensen, K. (2010). Active-passive calibration of optical tweezers in viscoelastic media. Review of Scientific Instruments, 81(1), 15103.

https://doi.org/10.1063/1.3280222

\section{General rights}

Copyright and moral rights for the publications made accessible in the public portal are retained by the authors and/or other copyright owners and it is a condition of accessing publications that users recognise and abide by the legal requirements associated with these rights.

- Users may download and print one copy of any publication from the public portal for the purpose of private study or research.

- You may not further distribute the material or use it for any profit-making activity or commercial gain

- You may freely distribute the URL identifying the publication in the public portal

If you believe that this document breaches copyright please contact us providing details, and we will remove access to the work immediately and investigate your claim. 


\title{
Active-passive calibration of optical tweezers in viscoelastic media
}

\author{
Mario Fischer, ${ }^{1}$ Andrew C. Richardson, ${ }^{2}$ S. Nader S. Reihani, ${ }^{3}$ Lene B. Oddershede, ${ }^{2}$ \\ and Kirstine Berg-Sørensen ${ }^{1}$ \\ ${ }^{1}$ Department of Physics, Technical University of Denmark, Kgs. Lyngby 2800, Denmark \\ ${ }^{2}$ Niels Bohr Institute, Copenhagen 2100, Denmark \\ ${ }^{3}$ Physics Department, Institute for Advanced Studies in Basic Sciences, P.O. Box 45195-1159, Zanjan, Iran
}

(Received 10 August 2009; accepted 9 December 2009; published online 14 January 2010)

\begin{abstract}
In order to use optical tweezers as a force measuring tool inside a viscoelastic medium such as the cytoplasm of a living cell, it is crucial to perform an exact force calibration within the complex medium. This is a nontrivial task, as many of the physical characteristics of the medium and probe, e.g., viscosity, elasticity, shape, and density, are often unknown. Here, we suggest how to calibrate single beam optical tweezers in a complex viscoelastic environment. At the same time, we determine viscoelastic characteristics such as friction retardation spectrum and elastic moduli of the medium. We apply and test a method suggested [M. Fischer and K. Berg-Sørensen, J. Opt. A, Pure Appl. Opt. 9, S239 (2007)], a method which combines passive and active measurements. The method is demonstrated in a simple viscous medium, water, and in a solution of entangled F-actin without cross-linkers. (C) 2010 American Institute of Physics. [doi:10.1063/1.3280222]
\end{abstract}

\section{INTRODUCTION}

Subcellular structures are collections of molecules in a complex, biological environment, which are in most cases out of equilibrium. Some of them perform time-dependent processes such as intracellular transport or cell division. Here, equilibrium energy states do not suffice for a quantitative description of the system and anyway, they could hardly be measured directly. The forces exerted in such processes can be measured more directly. The time and velocity dependencies of forces exerted between different sorts of biomolecules can give valuable information about the internal states of the molecules and about the interaction between the different types of molecules. This has been shown by in vitro force measurements on single molecules, whereby a better understanding of molecules such as motor proteins or fiber proteins has been obtained in the past two decades (Ref. 1 and references therein). Force measurements of biomolecules in their natural environment, the complex cell cytoplasm, would provide an even deeper understanding of their natural actions and interactions.

An optical trap is the only nanotool capable of manipulating biomolecules and organelles within the cytoplasm without perturbing the cellular membrane. Therefore, a single beam optical trap would be an optimal instrument to measure forces inside living cells. Normal calibration procedures for single beam optical traps ${ }^{2-4}$ apply in simple liquids only and often, one would need to know the viscosity, the index of refraction of the medium, and the size and shape of the probe. These are quantities that are often unknown when the probe is inside the cytoplasm or another viscoelastic medium. In the literature, previous investigations of probe particles manipulated by optical traps in viscoelastic media rely on ex situ calibration in a simple liquid such as water. ${ }^{5,6}$ Here, we demonstrate calibration in situ in a viscoelastic medium.
An optically trapped particle performs Brownian motion in a quasiharmonic potential. ${ }^{7}$ This motion can be recorded with high sampling rates by quadrant photo diode (QPD) position detection systems (Ref. 8 and references therein). Knowledge of the stiffness parameter $\kappa$ of the quasiharmonic potential and the position $x$ enables one to measure or exert prescribed forces of order $\mathrm{pN}$. In a calibration procedure the trapped particle is driven by forces of known characteristics and its trajectory is measured. The simplest driving force is the thermal stochastic force, causing a Brownian motion of the trapped particle. Such driving is denoted as passive. If the trapped object has a known shape and friction coefficient and if it is moving in a simple viscous fluid, force and position calibration is conveniently performed through power spectral analysis. ${ }^{2}$ Another type of calibration requires a controlled motion of the trapping laser with respect to the sample chamber. ${ }^{3,9}$ We denote this type of driving as active.

The Brownian motion of particles in viscoelastic media differs from that in viscous media. As a result, the power spectra of a bead in an actin gel ${ }^{10,11}$ and of a granule in a cell $^{12}$ deviate qualitatively from power spectra in viscous media such as glycerol or water. Also, if an endogenously occurring particle is used as handle for the optical trapping, its size and shape might not be known.

In this paper we show how to apply the so-called fluctuation-dissipation theorem (FDT) method ${ }^{13,14}$ to calibrate optical tweezers in a viscoelastic medium in a situation where the hydrodynamic interactions between the medium and the trapped particle are a priori unknown. The method combines passive and active measurements under conditions where the results of the FDT remain valid. Through the calibration we find the spring constant $\kappa$ that characterizes the strength of the optical trap, the positional calibration factor $\beta$, that relates voltages measured with the particle positions, the response function $\chi(f)$, the friction retardation spectrum 
TABLE I. Essential formulas. All quantities and equations are frequency dependent and have to be taken at the frequency $f=\omega /(2 \pi)$ of the stage/laser oscillations during the active part of the measurements. In the expression for the shear modulus, we have introduced the particle radius $r$.

\begin{tabular}{|c|c|c|c|}
\hline Quantity & Notation & \multicolumn{2}{|c|}{ Equal to } \\
\hline \multicolumn{4}{|c|}{ Undriven system } \\
\hline Power spectrum & $P$ & \multicolumn{2}{|c|}{$\lim _{T_{\text {meas }} \rightarrow \infty}\left\langle\left|\tilde{x}_{P}\right|^{2}\right\rangle / T_{\text {meas }}$} \\
\hline \multicolumn{4}{|c|}{ Driven system } \\
\hline Stage/laser amplitude & $A_{S / L}$ & $\begin{array}{l}\text { Stage driving } \\
\max \left\langle x_{S}\right\rangle\end{array}$ & $\begin{array}{l}\text { Laser driving } \\
\max \left\langle x_{L}\right\rangle\end{array}$ \\
\hline Stage/laser phase & $\phi_{S / L}$ & $\angle\left\langle x_{S}\right\rangle$ & $\angle\left\langle x_{L}\right\rangle$ \\
\hline Particle amplitude & $A_{P}$ & \multirow{2}{*}{\multicolumn{2}{|c|}{$\begin{array}{c}\max \left\langle x_{P}\right\rangle \\
\angle\left\langle x_{P}\right\rangle\end{array}$}} \\
\hline Particle phase & $\phi_{P}$ & & \\
\hline Phase shift & $\Delta \phi$ & $\Delta \phi=\phi_{S}-\phi_{P}$ & $\Delta \phi=\phi_{L}-\phi_{P}$ \\
\hline \multirow[t]{3}{*}{ Active spectrum } & $\tilde{R}_{S / L}$ & $\tilde{R}_{S}=\frac{A_{P}}{2 \pi f A_{S}} \times\{\sin [\Delta \phi]-i \cos [\Delta \phi]\}$ & $\tilde{R}_{L}=\frac{A_{P}}{2 \pi f A_{L}} \times\{-\sin [\Delta \phi]+i \cos [\Delta \phi]\}$ \\
\hline & & \multicolumn{2}{|l|}{ FDT method } \\
\hline & & Stage driving & Laser driving \\
\hline Trap stiffness (mass) & $\kappa(m)$ & $\kappa-\omega^{2} m=\frac{2 k_{B} T}{P} \operatorname{Re}\left\{\tilde{R}_{S}\right\}$ & $\kappa=\frac{2 k_{B} T}{P} \operatorname{Re}\left\{\widetilde{R}_{L}\right\}$ \\
\hline \multirow[t]{2}{*}{ Response function } & \multirow[t]{2}{*}{$\chi$} & $\underline{1-i \omega \widetilde{R}_{S}}$ & $i \omega \tilde{R}_{L}$ \\
\hline & & $\overline{\kappa-\omega^{2} m}$ & $-\frac{\omega \omega R_{L}}{\kappa}$ \\
\hline Frict. ret. spct. (mass) & $\tilde{\gamma}(m)$ & $\tilde{\gamma}=\widetilde{R}_{S} / \chi$ & $\tilde{\gamma}+i \omega m=-\frac{\kappa}{i \omega}\left(\frac{1}{i \omega \widetilde{R}_{L}}+1\right)$ \\
\hline Viscoelastic modulus (size, mass) & $G(r, m)$ & $\frac{i \omega}{6 \pi r} \tilde{\gamma}$ & $\frac{i \omega}{6 \pi r} \tilde{\gamma}$ \\
\hline \multirow{2}{*}{ Volt.-pos. conv. factor } & \multirow{2}{*}{$\beta$} & \multirow{2}{*}{$\frac{2 k_{B} T}{\omega P^{(\mathrm{volt})}} \cdot{\frac{\cot (\Delta \phi)}{\kappa^{\text {(volt) }}}}_{\text {a,b }}$} & 1 \\
\hline & & & $\overline{\operatorname{Re}\left\{\chi^{(\mathrm{volt})}\right\} \kappa^{(\mathrm{volt})}}$ \\
\hline
\end{tabular}

$\overline{\bar{a}}$ The quantities $P^{(\text {volt })}, \kappa^{\text {(volt) }}$, and $\chi^{(\text {volt) }}$ represent the power spectrum, the trap stiffness, and the response function obtained with the measured voltages $x_{P}^{\text {(volt) }}$ instead of positions $x_{P}$ in a natural length unit.

${ }^{\mathrm{b}}$ Equation is valid for low frequencies, $\omega \rightarrow 0$, and in media with vanishing static elasticity, $\lim _{\omega \rightarrow 0} \operatorname{Im}\{\widetilde{\gamma}(\omega)\} \rightarrow 0$.

${ }^{c}$ Equation is valid for low frequencies, $\omega \rightarrow 0$.

$\tilde{\gamma}(f)$, and for incompressible one-component fluids, also the shear modulus $G(f)$ of the viscoelastic medium in which the particle is immersed.

\section{THE FDT METHOD}

The theoretical background of the method is presented in Ref. 13. In this section we provide a recipe of the practical implementation of the method and thus, we repeat only the essentials to ensure a consistent description. Essential formulas are displayed in Table I, and below the calibration equations are restated as convenient formulations of the FDT.

In viscous media optical tweezers can be calibrated by applying a purely passive method, such as the power spectrum method. ${ }^{2}$ In this method, the power spectrum $P(\omega)$ of the Brownian motion of the trapped particle in equilibrium is determined from the Fourier transformed $\widetilde{x}(\omega)$ of the particle trajectory $x(t)$ which has been observed for a measurement time $T_{\text {meas }}$. In viscoelastic media, the power spectrum is analytically given by

$$
P(\omega)=\frac{2 k_{B} T \operatorname{Re}\{\widetilde{\gamma}(\omega)\}}{\left|\kappa+i \omega \widetilde{\gamma}(\omega)-\omega^{2} m\right|^{2}},
$$

where $m$ is the mass of the trapped particle. The quantity $\tilde{\gamma}(\omega)$ is called the friction retardation spectrum. Its real part accounts for dissipative processes, whereas its imaginary part accounts for elastic processes in the interaction between trapped particle and medium. For each frequency, Eq. (1) gives one equation for the unknowns $\kappa, \tilde{\gamma}(\omega)$, and $m$. Thus, additional information is required in order for us to solve for these unknowns if $m$ and the frequency dependence of $\widetilde{\gamma}(\omega)$ is unknown. The FDT method suggests obtaining that additional information by actively driving the system. Under the assumption that the FDT (Ref. 15)

$$
\chi^{\prime \prime}(\omega)=-\frac{\omega}{2 k_{B} T} P(\omega)
$$

holds for the viscoelastic medium and experimental situations considered, the information sought after is extracted from a combination of data from these active and passive measurement series.

The validity of the FDT requires that the active driving amounts to a perturbation of the equilibrium system only. In Eq. (2), $\chi^{\prime \prime}(\omega)$ represents the imaginary part of the response function. The response function is a quantity that describes nonequilibrium systems in linear response theory, and it relates external forces to average positions via

$$
\langle\widetilde{x}(\omega)\rangle=\chi(\omega) \widetilde{F}_{\text {ext }}(\omega) .
$$

Hence, $\chi(\omega)$ can be regarded as an inverse effective spring constant.

One way to apply an external perturbation to the system is to move the piezostage on which the sample is placed 
TABLE II. Explanation of quantities describing the system of a bead which is trapped by optical tweezers in a viscoelastic medium in linear response theory.

\begin{tabular}{lll}
\hline \hline Quantity & Notation & \multicolumn{1}{c}{ Physical content } \\
\hline Particle mass & $m$ & $\begin{array}{l}\text { Particle property; mass of the particle trapped by the optical } \\
\text { tweezers. }\end{array}$ \\
Trap stiffness & $\kappa$ & $\begin{array}{l}\text { Interaction property; spring constant of the harmonic potential } \\
\text { exerted by the optical tweezers. }\end{array}$ \\
Friction retardation \\
spectrum
\end{tabular}

(stage driving). If the sample container is sealed on all sides, the liquid in which the trapped particle is immersed can be assumed to be comoving with the piezostage. In that case, one can formulate the FDT in a more convenient way. We introduce the active spectrum for stage driving $\widetilde{R}_{S}(\omega)$

$$
\widetilde{R}_{S}(\omega):=\tilde{\gamma}(\omega) \chi(\omega)=\frac{\langle\widetilde{x}(\omega)\rangle}{i \omega \tilde{x}_{S}(\omega)} .
$$

The stage follows a chosen trajectory $x_{S}(t)$ that, at least in principle, is infinitesimal around $x_{S}=0$ in order to provide the external perturbation. By reformulating the FDT one obtains, equivalently to Eq. (2)

$$
\left(\kappa-\omega^{2} m\right)_{\omega}=\frac{2 k_{B} T}{P(\omega)} \operatorname{Re}\left\{\widetilde{R}_{S}(\omega)\right\} .
$$

Equation (5) is still the FDT that in this form represents a general measurement prescription for finding the unknowns $\kappa$ and $m$. Note that $P(\omega)$ in Eq. (5) still represents the power spectrum of the unperturbed system and is therefore obtained in the passive part of the measurements. Simulation results and analytical considerations suggest that for a calibration, it is most practical and reliable to perturb the system with harmonic oscillations. ${ }^{13}$ In the special case of a sinusoidally moved stage, $x_{S}(t)=A_{S} \sin \left(\omega_{S} t+\phi_{S}\right)$, the trapped particle responds with sinusoidal motion, $\left\langle x_{P}(t)\right\rangle=A_{P} \sin \left(\omega_{S} t+\phi_{P}\right)$. Equation (5) then becomes

$$
\left(\kappa-\omega^{2} m\right)_{\omega_{S}}=\frac{2 k_{B} T}{P\left(\omega_{S}\right)} \frac{A_{P}}{\omega_{S} A_{S}} \sin (\Delta \phi),
$$

where $\Delta \phi$ is the phase difference between stage and particle oscillations, $\Delta \phi=\phi_{S}-\phi_{P}$. Equation (6) represents a simple formula for the experimental determination of the trap stiffness, since all magnitudes on the right-hand side can be obtained from measurements. Parameter values for $\kappa$ and $m$ may be found by fitting the parabolic function on the left-hand side of Eqs. (5) and (6) to the experimental values.

Another option to drive the system is to relocate the trap center (laser driving). Moreover, using this application of the FDT to obtain the trap stiffness $\kappa$ would allow other parameters of the linear system of the trapped particle in the viscoelastic medium to be found without any further measurements. These parameters are the response function $\chi(\omega)$, the friction retardation spectrum $\widetilde{\gamma}(\omega)$, and the voltage-position conversion factor $\beta:=x_{P}^{\text {(volt) }} / x_{P}$ which relates measured voltages $x_{P}^{\text {(volt) }}$ to actual particle positions $x_{P}$. All quantities describing the system of a trapped bead in a viscoelastic medium in linear response theory are summarized in Table II. The equations for determining the parameters for both stage and laser driving are shown in Table I.

\section{MATERIALS AND METHODS}

\section{A. Experiments}

In principle, the experimental procedure consists of two parts, involving active and passive time series measurements. For technical reasons, since the active part in our setup is performed with stage driving, three additional steps are required: direct positional calibration, pixel calibration, and stage-bead phase delay calibration. The entire procedure is sketched in Fig. 1 and explained in more detail below.

The optical tweezers setup consists of a neodymium doped yttrium orthvanadate $\left(\mathrm{Nd}: \mathrm{YVO}_{4}\right)$ laser operating at 1064 nm (10 W, Spectra Physics Millenia) which is directed into an inverted microscope (Leica DMIRBE), equipped with an oil objective with a numerical aperture of 1.32 (Leica HCX PL APO 63x/1.32, oil). A QPD (S5981, Hamamatsu) positioned in the conjugate position of the back focal plane of the condenser and a charge-coupled device (CCD) camera (Sony XC-ES50, $25 \mathrm{~Hz}$ ) mounted on the side port of the 
a) Force calibration (FDT method), passive part

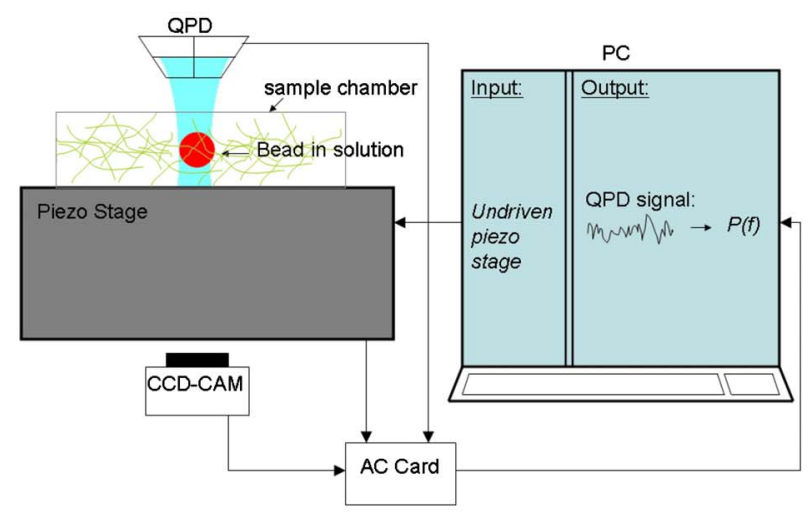

c) Direct positional calibration (sinusoidal stage driving)

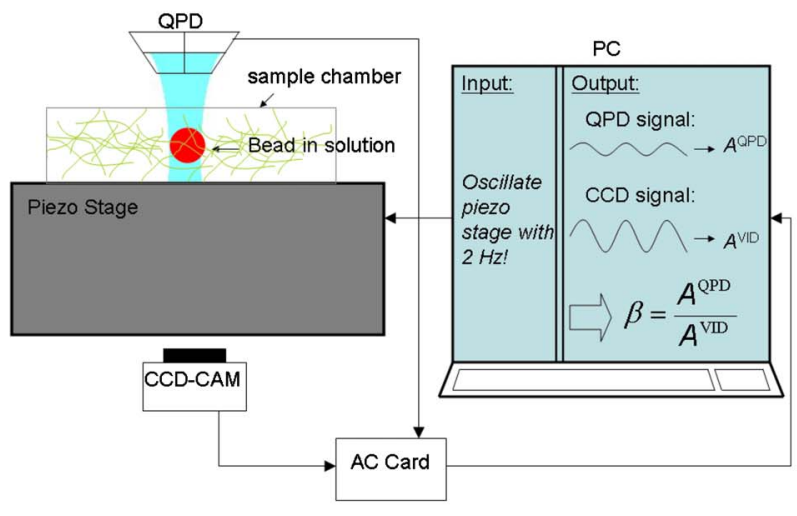

b) Force calibration (FDT method), active part

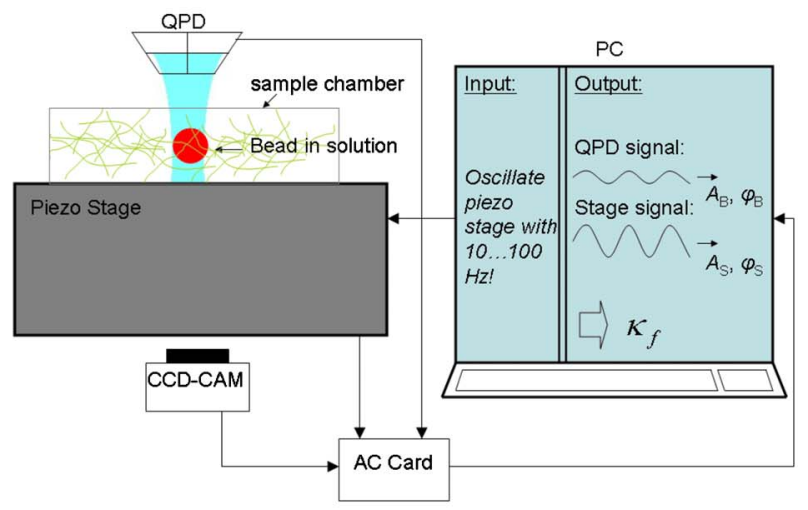

d) Pixel Size Calibration (displacement of stage by a known distance)

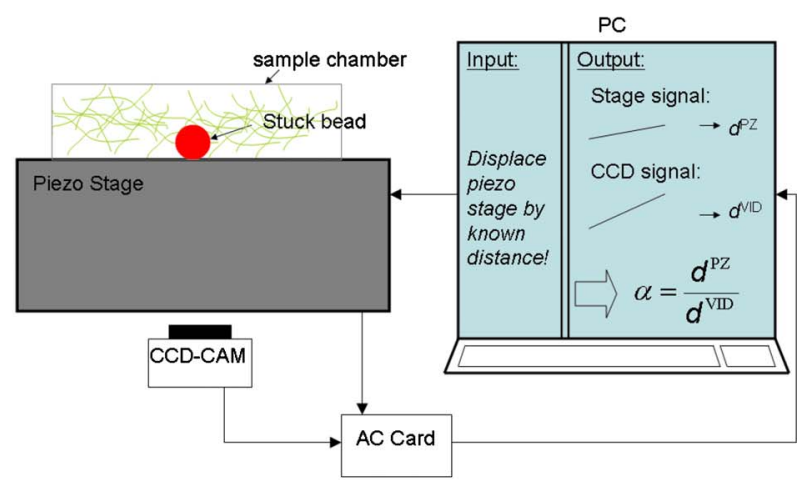

e) Phase Correction Calibration for calibrating delay between $\mathrm{AC}$ card channels (sinusoidal stage driving)

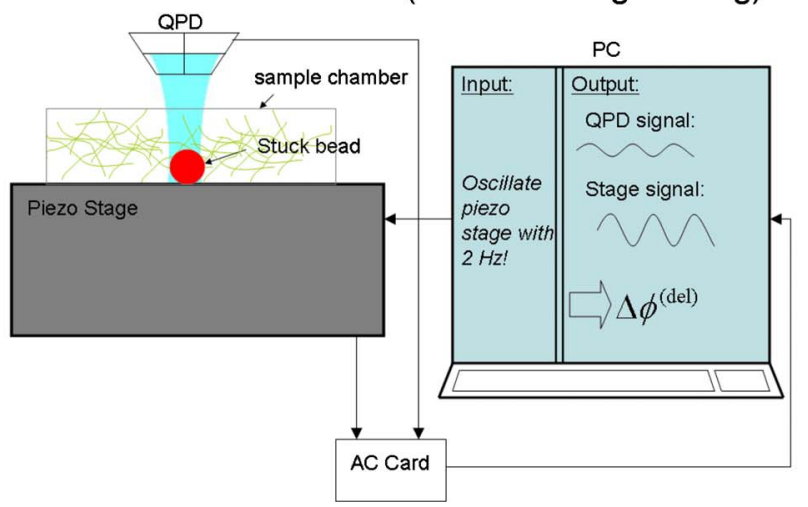

FIG. 1. (Color online) Sketch of the different steps in the experimental procedure. In (a)-(c), the bead is in solution and follows the oscillations of the stage with a delay. In (d) and (e), the bead is bound to the coverslip and follows the oscillations of the stage precisely.

microscope provide two different means of detecting the trapped bead. Further details of the setup may be found in Ref. 16.

The hardware enables simultaneous acquisition of bead and stage positions which is necessary for determining the phase shift $\Delta \phi(\omega)$ between bead and stage. Bead and stage positions were acquired with a fast data acquisition card [NI PCI-MIO-16E-4 (old model designation) and NI PCI-6040E (new model designation)]. Home-made LABVIEW programs (LABVIEW 7.1 and 8.5, National Instruments) were used to acquire data from the photodiode and the CCD camera to move the sample chamber (mounted on the pi- ezostage) in three dimensions (3D) and analyze the images obtained.

A sample chamber was prepared from two cover slips with double sided sticky tape in between as spacer. For the experiments in water, polystyrene beads of a diameter of $1.65 \mu \mathrm{m}$ were diluted in millipore water $\left(1 / 10^{6}\right)$, injected into a chamber and sealed with silicon/vacuum grease. The actin network was created by adding $450 \mu \mathrm{l}$ of G-buffer (2.0 mM Tris $\mathrm{HCl}, 0.2 \mathrm{mM}$ ATP, $0.2 \mathrm{mM} \mathrm{CaCl}$, and $0.5 \mathrm{mM}$ DTT) to $1 \mathrm{mg}$ of freeze dried actin (Sigma/from rabbit muscle) as well as $5 \mu \mathrm{l}$ of bead solution $(2.1 \mu \mathrm{m}$, polystyrene beads (Spherotech) at 1/250 bottle concentration) and 
$45 \mu \mathrm{l}$ of F-buffer $(750 \mathrm{mM} \mathrm{KCl}, 20 \mathrm{mM} \mathrm{MgCl} 2,10 \mathrm{mM}$ ATP, and $4 \mathrm{mM}$ Tris $\mathrm{HCl}$ ), giving a final actin concentration of $2 \mathrm{mg} / \mathrm{ml} .{ }^{17}$ Preparation was done on ice. Thereafter, the Eppendorf tube was repeatedly tilted to mix the sample contents before approximately $30 \mu \mathrm{l}$ of it was transferred to the prepared sample chamber and sealed with silicon grease. The chamber then rotated in the refrigerator for at least $1 \mathrm{~h}$ before the experiments were undertaken.

\section{Passive measurements}

Passive measurements are illustrated in Fig. 1(a). A bead was trapped with the optical tweezers at a fixed distance $l$ from the microscope coverslip with $l$ ranging between 5 and $23 \mu \mathrm{m}$. The bead motion was recorded with the QPD. The power of the laser was kept constant during the course of the calibration, typically at $110-120 \mathrm{~mW}$ at the exit of the laser, with an estimated intensity of $\sim 15 \mathrm{~mW}$ at the sample. The Brownian motion of the trapped bead was sampled at $10 \mathrm{kHz}$ whereby $N_{\text {wind }}$ time series were recorded. The power spectra from each of these windows were calculated. In order to check whether changes occurred because of the active driving, passive measurements were performed in between active ones.

\section{Active measurements}

Figure 1(b) illustrates the active measurements. The system was driven by sinusoidally oscillating the piezostage in the $x$ direction with seven different frequencies between 10 and $70 \mathrm{~Hz}$, for the experiments in water and with 19 different frequencies between 10 and $100 \mathrm{~Hz}$, for measurements in the actin solution. Each time the stage was moved for $10 \mathrm{~s}$ and the bead motion was recorded with a sampling frequency of $2 \mathrm{kHz}$. For measurements in water, the driving amplitudes were chosen as described below. For measurements in actin, we applied a driving amplitude of $200 \mathrm{~nm}$. The stage motion was recorded with the inbuilt stage position monitoring system.

\section{Direct positional calibration}

The direct positional calibration is illustrated in Fig. 1(c). Direct positional calibration is used to determine the conversion factor $\beta$ which translates the output voltages of the photodiode to real positions through a measurement independent from the active and passive measurement series. We denote this value $\beta^{(\mathrm{SIN})}$. The stage was driven sinusoidally at a fixed frequency and measurements from the QPD were compared with measurements based on the images taken by the CCD camera. The QPD facilitates the measurement of spatial displacements as small as $1 \mathrm{~nm}$ with a temporal resolution of MHz. ${ }^{18,19}$ However, the linearity of the conversion factor $\beta$, which translates the output voltages of the photodiode to positions in a natural length unit is limited to displacements below $40 \%$ of the radius of the trapped bead. ${ }^{20}$ On the contrary, a CCD detection scheme has the advantage of remaining linear for a wider range of displacements, provided that the objective is corrected for field curvature aberration. However, this method suffers from lower temporal resolution, as limited by the frame rate of the cam- era. The spatial resolution is limited by pixel size but may be improved simply by zooming in on the objects in the field of view. In our case, we zoomed in on the trapped bead with a diameter of $2 \mu \mathrm{m}$ to achieve a pixel size on the order of $12 \mathrm{~nm}$, calibrated as described below.

\section{Pixel calibration}

The pixel size calibration is illustrated in Fig. 1(d). From the images, 3D detection of the bead position was based on the brightest ring in the image of the bead. We applied a binary mapping with a threshold value to transform the picture into a bright ring on a black background. Then the center of the ring was interpreted as the lateral position of the bead while its diameter was used to calculate the axial position as follows: A stuck bead was moved in predefined steps of length $d^{\mathrm{PZ}}$, in both lateral and axial directions using the piezostage while grabbing images of the bead. The resulting images underwent binary mapping and were then analyzed to extract precise values of pixel sizes in both lateral and axial directions, $d^{\mathrm{VID}}$, and the conversion factor $\alpha$ was determined from the ratio. Further details may be found in Ref. 7 .

\section{Phase correction calibration}

Calibration of the phase correction is illustrated in Fig. 1(e). The acquisition of bead position is delayed relative to the determination of the stage position, with a delay time $t^{(\mathrm{del})}$. Thus, for the correct determination of $\Delta \phi$ in Eq. (6), we must correct for the phase delay $\Delta \phi^{(\mathrm{del})}=t^{(\mathrm{del})} f_{S}$

$$
\Delta \phi^{(\mathrm{corr})}=\Delta \phi^{(\mathrm{fit})}-\Delta \phi^{(\mathrm{del})} .
$$

Here, $\Delta \phi^{(\text {fit })}$ was found from fits of sinusoidal functions to the bead and stage positions in the active measurements. For determination of the phase delay $\Delta \phi^{(\mathrm{del})}$, we made an additional experiment using a bead stuck to the coverslip. We recorded the stage signal and the QPD signal of the stuck bead while sinusoidally oscillating the stage with a frequency of $2 \mathrm{~Hz}$. Since the stuck bead moves exactly like the stage, the phase difference between the phases in sinusoidal fits to stage and bead signal gives the time lag due to the data acquisition process. We obtained $t^{(\mathrm{del})}=494 \pm 17 \mu \mathrm{s}$. In principle, the phase correction calibration should be performed only once for a given setup, with a given data acquisition card.

\section{SELECTION OF OSCILLATION AMPLITUDE}

Here, the choice of deflection amplitude $A_{L / S}$ is discussed for both laser and stage driving while, in our experiments only stage driving has been applied. The larger the deflection amplitude of the laser/stage, the larger is the deflection of the trapped particle and the better is the signalnoise ratio after averaging. On the other hand, the deflection $A_{L / S}$ has to be chosen relatively small due to several constraints. First, the bead should stay in the harmonic trapping region during deflection of the laser or stage to ensure a linear response of the trap. Second, the center of the bead must remain within the linear detection range of the QPD. Third, since the method is based on the FDT, the laser/stage motion should generate a small perturbation of the equilib- 


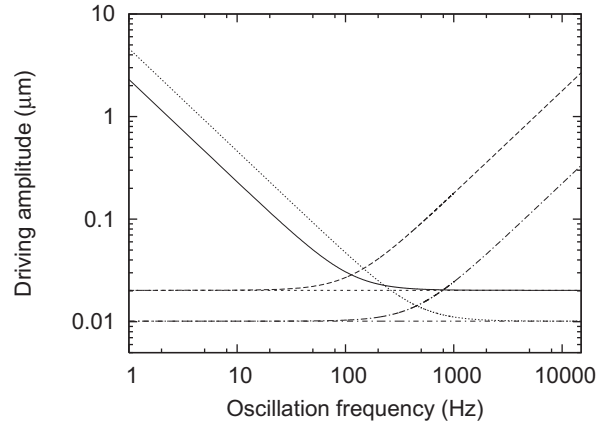

FIG. 2. Required stage and laser amplitudes for obtaining particle oscillations with an amplitude equal to the half width of the trap for two different spring constants $\kappa$. Solid line: stage amplitude for $\kappa=0.01 \mathrm{pN} / \mathrm{nm}$; Dashed line: laser amplitude for $\kappa=0.01 \mathrm{pN} / \mathrm{nm}$; Short-dashed line: half width of trap when $\kappa=0.01 \mathrm{pN} / \mathrm{nm}$. Dotted line: stage amplitude for $\kappa=0.04 \mathrm{pN} / \mathrm{nm}$; Long-dashed-dotted line: laser amplitude for $\kappa=0.04 \mathrm{pN} / \mathrm{nm}$; Short-dashed-dotted line: half width of trap when $\kappa=0.04 \mathrm{pN} / \mathrm{nm}$. The corresponding corner frequencies are $f_{c}=114$ and $455 \mathrm{~Hz}$, respectively. The particle is assumed to be a spherical bead of radius $R=875 \mathrm{~nm}$ and density $\rho=1.05 \mathrm{~g} / \mathrm{cm}^{3}$ which moves in water with viscosity $\eta=0.0009 \mathrm{~Pa}$ s. Hydrodynamic interactions beyond simple Stokes friction are neglected.

rium system, i.e., the amplitude $A_{L / S}$ should be as low as possible. This third constraint is apparently the most stringent one. Since one has to choose some finite value, a reasonable choice would be to set $A_{S / L}$ so that the bead amplitude $A_{B}$ would be equal to the half width of the trap, $\left(k_{B} T / \kappa\right)^{1 / 2}$. That choice should still ensure a linear response. We can now use the response function $\chi(\omega)$ to calculate the required stage/laser amplitudes for sinusoidal driving with angular frequency $\omega_{K}$ according to the laser/stage trajectory

$$
x_{L / S}(t)=A_{L / S} \sin \left(\omega_{K} t\right) .
$$

One has Eq. (3), with the external force from the laser/stage being

$$
\begin{aligned}
& \tilde{F}_{L}(\omega)=\frac{\kappa}{i \omega} i \omega \tilde{x}_{L}(\omega), \\
& \tilde{F}_{S}(\omega)=\widetilde{\gamma}(\omega) i \omega \tilde{x}_{S}(\omega) .
\end{aligned}
$$

By taking the norm of Eq. (3), inserting the laser/stage velocity spectrum for sinusoidal driving and requiring for the bead amplitude $A_{B}\left(\omega_{K}\right) \equiv\left|\left\langle\widetilde{x}\left(\omega_{K}\right)\right\rangle\right| \stackrel{!}{=}\left(k_{B} T / \kappa\right)^{1 / 2}$, one obtains

$$
\begin{aligned}
& A_{S}\left(\omega_{K}\right)=\frac{1}{\left|\chi\left(\omega_{K}\right)\right|\left|\omega_{K} \tilde{\gamma}\left(\omega_{K}\right)\right|} \sqrt{\frac{k_{B} T}{\kappa}}, \\
& A_{L}\left(\omega_{K}\right)=\frac{1}{\left|\chi\left(\omega_{K}\right)\right| \kappa} \sqrt{\frac{k_{B} T}{\kappa}} .
\end{aligned}
$$

Those two results are plotted over the frequency $f_{K}=\omega_{K} /(2 \pi)$ for different spring constants $\kappa$, in Fig. 2, for a polystyrene bead with radius $R=875 \mathrm{~nm}$ and density $\rho=1.05 \mathrm{~g} / \mathrm{cm}^{3}$ in a simple viscous medium of viscosity $\eta=0.0009$ Pa s.

Note that the curves plotted in Fig. 2 are from analytical considerations. In reality, state-of-the-art stages can only be oscillated up to frequencies on the order of $100 \mathrm{~Hz}$. For laser driving one must keep in mind that the harmonic trapping region has a finite extension, so for a linear response the laser oscillation amplitude should not exceed a value that is half of that extension. Note further, the difference between stage and laser driving in Fig. 2: While high stage position amplitudes are necessary at low stage driving frequencies, high laser position amplitudes are required at high laser driving frequencies. The physical reason for that is that the interaction between the particle and the laser trap is proportional to their relative positions but the coupling between particle and a simple viscous medium is only via their relative velocities. Thus at low frequencies when the stage is only moving slowly, one needs a high stage amplitude to deflect the bead from its equilibrium position and vice versa, at high frequencies. In addition, note that the crossover frequency between the curves for required stage and laser amplitudes in Fig. 2 is very close to the corner frequency $f_{c}=\kappa /\left(2 \pi \gamma_{0}\right)$.

To find driving amplitudes $A_{S}\left(\omega_{K}\right)$ required for our experiments with stage oscillations, Eq. (11) has been applied by using estimates for the parameters $\kappa, \widetilde{\gamma}(\omega)$, and $\chi(\omega)$. In experiments in general viscoelastic media, these parameters are unknown before the calibration. Then, Eqs. (11) and (12) cannot be applied a priori for the calculation of the required laser or stage amplitude $A_{L / S}\left(\omega_{K}\right)$. These amplitudes rather have to be adjusted in a way that the response of the particle exhibits an amplitude that is roughly equal to the observed half width of the trap.

\section{A. Data handling}

All of the previous equations were given in frequency space. In practice, data are recorded as positions $x(t)$ and $y(t)$ for a single trapped particle for a finite measurement time $T_{\text {meas }}$ with a finite sampling rate $f_{\text {sample. }}$. Such data sets have to be Fourier transformed whereby one obtains discrete frequency-dependent transforms $\hat{x}_{k}$ at the frequencies $f_{k}=k / T_{\text {meas }}, k=-T_{\text {meas }} f_{\text {sample }} / 2+1, \ldots, T_{\text {meas }} f_{\text {sample }} / 2$

$$
\hat{x}_{k}=\delta t \sum_{j}^{N} e^{-i 2 \pi j k / N} x\left(t_{j}\right)
$$

with $t_{j}=j \delta t, \delta t=1 / f_{\text {sample }}$, and $N \delta t=T_{\text {meas }}$.

From the data measured during the passive part with the QPD, the Fourier transformed data $\hat{x}_{k}^{\text {(volt) }}$ lead to the power spectrum $P_{k}^{(\text {volt })}$. From windowing by averaging over $N_{\text {wind }}=10$ data sets and blocking by averaging over $N_{\text {block }}=20$ adjacent frequencies, one obtains the windowed and blocked power spectrum ${ }^{2}$

$$
\overline{\left\langle P_{k}^{(\text {volt })}\right\rangle_{\text {wind }}}=\frac{\overline{\left\langle\left.\hat{x}_{k}^{(\text {volt })}\right|^{2}\right\rangle_{\text {wind }}}}{T}
$$

to be combined with the measurements from the active part.

\section{RESULTS AND DISCUSSION}

\section{A. Experiments in water}

The first series of experiments to validate the FDT method were experiments on beads trapped in a simple liquid (water) with known viscous properties. These sets of experiments allow for a direct comparison with established methods, i.e., the power spectrum (PSD) method. ${ }^{2}$ 


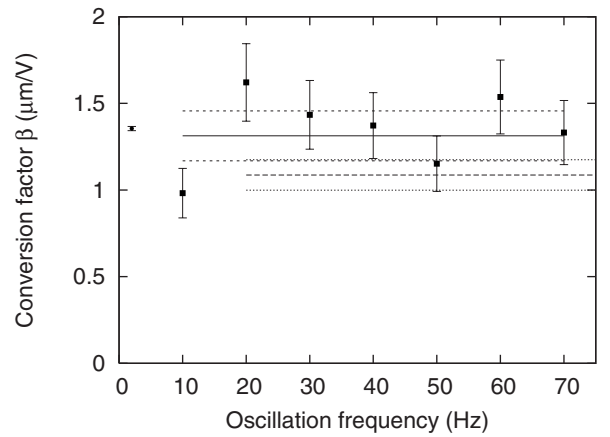

FIG. 3. Voltage-position conversion factor values obtained with three different methods, measurements in water. The long-dashed line indicates the value $\beta^{(\mathrm{PSD})}$ obtained with the PSD method as described in the text. The dotted lines indicate $\beta^{(\mathrm{PSD})} \pm$ two times the standard deviation. One standard deviation corresponds to a relative error of $4.1 \%$. Solid squares with error bars show the frequency-dependent values $\beta_{K}^{(\mathrm{FDT} \text {,ex })}$ that result from calibration with the FDT method. The relative error of $\sim 14 \%$ originates from statistical errors and errors from the uncertainty of the temperature. The average, $\beta^{\text {(FDT,av) }}$, is shown as a solid line, with short-dashed lines indicating $\beta^{\text {(FDT,av) }} \pm$ two times the standard deviation (one standard deviation gives a relative error of $5.5 \%$ ). Finally, the solid circle with error bars indicates the conversion factor $\beta^{(\mathrm{SIN})}$ as obtained by the independent positional calibration.

\section{Application of PSD method}

When applying the PSD method, ${ }^{2}$ it is sufficient to evaluate the undriven data from the bead immersed in water. Application of the PSD method results in values for two fitted parameters, the corner frequency $f_{c}^{(\mathrm{PSD})}$ and the diffusion coefficient $D^{\text {(PSD,volt) }}$. Conversion of the diffusion coefficient to metric units provides the factor $\beta$ as discussed below. The trap stiffness is obtained according to

$$
\kappa^{(\mathrm{PSD})}=2 \pi \gamma_{0}^{*} f_{c}^{(\mathrm{PSD})}
$$

with the friction coefficient

$$
\gamma_{0}^{*}=6 \pi \eta R\left(1+\frac{9 R}{16 l}\right)
$$

where the finite distance $l=5 \mu \mathrm{m}$ between bead and microscope coverslip has been accounted for. With $\eta\left(\vartheta=24.5^{\circ} \mathrm{C}\right)=0.0009 \mathrm{~Pa} \mathrm{~s}$, one obtains from averaging over the $N_{\text {wind }}=20$ data sets

$$
\kappa^{(\mathrm{PSD}, \mathrm{av})}=(46.1 \pm 1.1) \mathrm{pN} / \mu \mathrm{m} .
$$

The temperature $\vartheta$ enters this result indirectly because the viscosity is temperature dependent. The error contribution arising from the temperature uncertainty is $2.2 \%$ and has been obtained by assuming that the temperature at the bead position is known with an uncertainty of $\sim 1 \mathrm{~K} .{ }^{21}$ At $\vartheta=25{ }^{\circ} \mathrm{C}$, the viscosity changes by $\sim 20 \mu \mathrm{Pa} \mathrm{s}$ per kelvin which corresponds to a relative error of $2.2 \%$. The conversion factor $\beta$ is determined with the PSD method from the relation

$$
\beta^{(\mathrm{PSD})}=\sqrt{D / D^{(\mathrm{PSD}, \mathrm{volt})}}
$$

with the theoretical value for the diffusion coefficient $D=k_{B} T / \gamma_{0}^{*}$. The resulting value is displayed in Fig. 3 .

\section{Application of the FDT method}

The experimental setup available allowed for stagedriving only, and below we describe our results when conducting experiments with that driving procedure.

\section{Positional calibration}

The conversion factor $\beta$ estimated from data from active measurements $\beta^{(\mathrm{FDT}, \mathrm{ex})}$ is found through the discretized version of the expression stated in Table I

$$
\beta_{K}^{(\mathrm{FDT}, \mathrm{ex})}=\frac{2 k_{B} T}{2 \pi f_{K} \overline{\left\langle P_{K}^{(\text {volt })}\right\rangle_{\text {wind }}}} \cdot \frac{\cot \left[\Delta \phi_{K}^{(\text {corr })}\right]}{\kappa_{K}^{\text {(FDT,av,volt) }}} .
$$

Since we measure in water at low frequencies, a value with lower statistical error may be obtained by averaging

$$
\beta^{(\mathrm{FDT}, \mathrm{av})}=\left\langle\beta_{K}^{(\mathrm{FDT}, \mathrm{ex})}\right\rangle_{K} .
$$

The results for $\beta_{K}^{(\mathrm{FDT}, \mathrm{ex})}$ and $\beta^{(\mathrm{FDT}, \mathrm{av})}$ are plotted in Fig. 3 . Furthermore, the results from the PSD method, $\beta^{\text {(PSD) }}$, Eq. (18), and the estimate from the independent method described in Sec. III, $\beta^{(\mathrm{SIN})}$, are given. As seen in the figure, we obtain good agreement between the results from the FDT method and the independent method, whereas the PSD method yields a somewhat lower result. We suspect mechanical drift to be the cause for this discrepancy. In the experiments described here, we work in a very narrow frequency range at quite low frequencies, and application of the PSD method is thus more susceptible to errors due to drift than in a typical optical tweezers experiment.

\section{Trap stiffness}

For calibration with the FDT method, the discretized version of the basic equation in terms of amplitudes and angles, Eq. (6), is applied

$$
\kappa_{K}^{(\mathrm{FDT}, \text { volt) }}=\frac{2 k_{B} T}{\left\langle P_{K}^{(\text {volt })}\right\rangle_{\text {wind }}} \cdot \frac{A_{P, K}^{(\text {fit,volt) }}}{2 \pi f_{K} A_{S, K}^{(\text {fit,ex) }}} \sin \left[\Delta \phi_{K}^{\text {(corr) }}\right],
$$

where the capital $K$ is the index of the driving frequency $f_{K}$ of the laser/stage and the mass term has been neglected. This result has units $\mathrm{pN} /\left(\right.$ volt unit). For obtaining a result $\kappa_{K}^{\text {(ex) }}$ which has units $\mathrm{pN} / \mathrm{nm}$, it has to be divided by the conversion factor $\beta^{\text {(SIN) }}$

$$
\kappa_{K}^{(\mathrm{FDT}, \mathrm{ex})}=\frac{\left[\kappa-(2 \pi f)^{2} m\right]_{K}^{(\text {volt })}}{\beta^{(\mathrm{SIN})}} .
$$

Experimental spectra display statistical noise. Therefore it is appropriate to average those results to obtain a value with lower statistical error

$$
\kappa^{(\mathrm{FDT}, \mathrm{av})}=\left\langle\kappa_{K}^{(\mathrm{FDT}, \mathrm{ex})}\right\rangle_{K} .
$$

The results for $\kappa_{K}^{(\mathrm{FDT}, \mathrm{ex})}$ and $\kappa^{(\mathrm{FDT}, \mathrm{av})}$ are plotted in Fig. 4 together with the result from the PSD method, Eq. (17).

For $\kappa^{(\mathrm{FDT}, \mathrm{av})}$ one obtains

$$
\kappa^{(\mathrm{FDT}, \mathrm{av})}=(43.6 \pm 1.3) \mathrm{pN} / \mu \mathrm{m},
$$

a result with a total relative error of $4 \%$. Thus, within their error bars, the calibration results from the PSD method and the FDT method agree. 


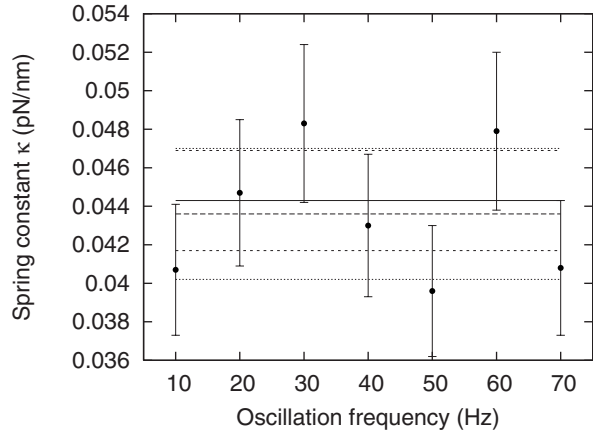

FIG. 4. Trap stiffness values obtained with two different calibration methods, measurements in water. The long-dashed line shows $\kappa^{(\mathrm{PSD}, a v)}$, Eq. (17). It is obtained from a fit ranging up to $2 \mathrm{kHz}$. The average value \pm two times its relative error of $3.3 \%$ is shown with dotted lines. Data points with error bars indicate $\kappa_{K}^{\text {(FDT,ex) }}$, the relative errors of $\sim 8.5 \%$ includes statistical errors, errors from the positional calibration, and the uncertainty of the temperature. The seven values for $\kappa_{K}^{(\mathrm{FDT}, \mathrm{ex})}$ exhibit roughly overlapping error bars and therefore all values are included in the estimate $\kappa^{\text {(FDT,av) }}$, indicated by the solid line. Short-dashed lines give $\kappa^{(\mathrm{FDT}, \mathrm{av})} \pm$ two times the relative error of $4.0 \%$.

\section{Response function}

The response function at frequency $f_{K}$ is obtained as

$$
\chi_{K}^{(\mathrm{FDT}, \mathrm{ex})}=\frac{1-i 2 \pi f_{K} \hat{R}_{S, K}^{(\mathrm{ex})}}{\kappa^{(\mathrm{FDT}, \mathrm{av})}} .
$$

The real and imaginary parts of the active spectrum which are necessary for that computation are obtained by using the relations

$$
\begin{aligned}
& \operatorname{Re}\left\{\hat{R}_{S, K}^{(\mathrm{ex})}\right\}=\frac{A_{P, K}^{(\mathrm{fit}, \mathrm{volt})}}{2 \pi f_{K} A_{S, K}^{(\text {fit,ex) }} \beta^{(\mathrm{SIN})}} \sin \left[\Delta \phi^{(\mathrm{corr})}\right], \\
& \operatorname{Im}\left\{\hat{R}_{S, K}^{(\mathrm{ex})}\right\}=-\frac{A_{P, K}^{(\text {fit, volt) }}}{2 \pi f_{K} A_{S, K}^{(\mathrm{fit}, \mathrm{ex})} \beta^{(\mathrm{SIN})}} \cos \left[\Delta \phi^{(\mathrm{corr})}\right] .
\end{aligned}
$$

The experimental result for the response function agrees well with the expected response function (data not shown)

$$
\chi(f)=\frac{1}{\kappa^{(\mathrm{FDT}, \mathrm{av})}+i 2 \pi f \gamma_{0}^{*}} .
$$

\section{Friction retardation spectrum}

As the next step, the friction retardation spectrum is determined from

$$
\hat{\gamma}_{K}^{(\mathrm{FDT}, \mathrm{ex})}=\hat{R}_{S, K}^{(\mathrm{ex})} / \chi_{K}^{(\mathrm{FDT}, \mathrm{ex})}
$$

by using the results from the calculation of the response function from the last paragraph. The result for the real part is plotted in Fig. 5(a). The data values lie roughly on a constant line given by $\gamma_{0}^{*}$. Thus, good agreement is achieved with the model for friction in the proximity of a hard wall which, for simple Stokes friction, is frequency independent to the first order. The result for the imaginary part is plotted in Fig. 5(b). The imaginary part of $\tilde{\gamma}$ represents elastic contributions to the friction force and it is small compared to the real part. The data are not inconsistent with the theoretical curve $\operatorname{Im}\left\{\widetilde{\gamma}_{\text {hw }, R / l}\right\}(f)$ from Ref. 2 [Eq. (33)]. The statistical errors of the data points are very large, however, and a clear

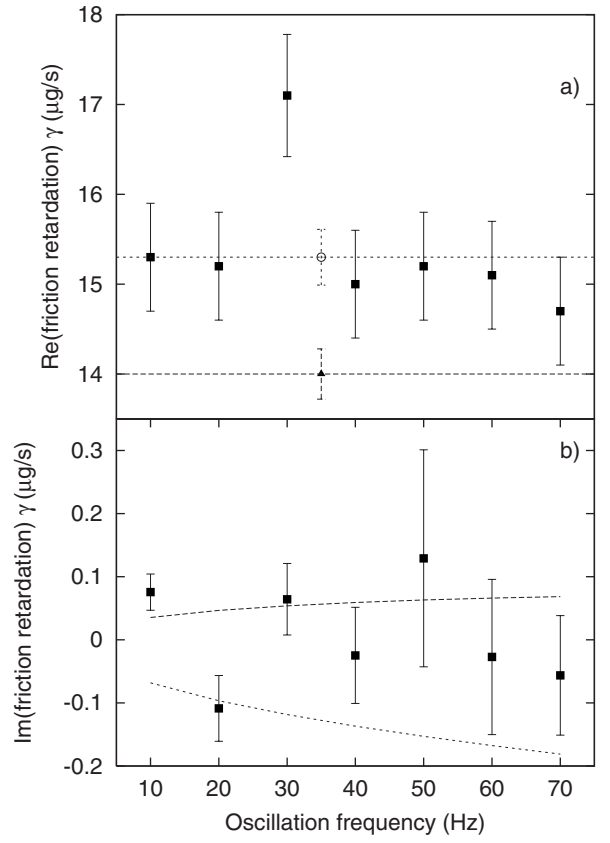

FIG. 5. Measurements in water. (a) Real part of the friction retardation spectrum $\operatorname{Re}\left\{\hat{\gamma}_{K}^{\text {(FDT,ex) }}\right\}$, shown as solid squares with error bars. For comparison to expectations, the long-dashed line shows $\gamma_{0}=14.0 \mathrm{ng} / \mathrm{s}$, corresponding to the bulk Stokes friction. The filled triangle with (long-dashed line) error bars indicates the $2 \%$ error due to the uncertainty in temperature. The dotted line indicates $\operatorname{Re}\left\{\widetilde{\gamma}_{\mathrm{hw}, R / l}\right\}(f)=\gamma_{0}^{*}=15.3 \mathrm{ng} / \mathrm{s}$, including corrections due to the finite distance $l$ to the coverslip, to first order in $R / l$. The open circle with (dotted line) error bars indicates uncertainty due to temperature. This demonstrates that the FDT method correctly reproduces the effect of increased viscosity due to the proximity of the microscope coverslip. (b) Imaginary part of the friction retardation spectrum $\operatorname{Im}\left\{\hat{\gamma}_{K}^{(\text {FDT,ex })}\right\}$, shown as filled squares with error bars. The long-dashed line shows the imaginary part of the friction retardation spectrum in proximity to a hard wall, $\operatorname{Im}\left\{\widetilde{\gamma}_{\mathrm{hw}, R / l}\right\}(f)$ (Ref. 2) [Eq. (33)], for $R / l=825 / 5000$. For comparison, the imaginary part of Stokes friction (Ref. 2) [Eq. (31)], is plotted as a dotted line.

assessment is not possible. Furthermore, systematic errors could play a significant role here. In order to obtain experimental data with smaller error bars, the measuring time must be chosen longer, especially in the undriven case. Furthermore, if measurements were performed for a bead closer to the microscope coverslip then the imaginary part of the friction retardation spectrum should become larger, which would reduce the relevance of both statistical and systematic errors.

\section{B. Experiments in F-actin}

The second series of experiments to validate the FDT method were experiments on beads trapped in a semidilute solution of F-actin without cross-linkers. Thus, we also test the method in a true viscoelastic medium, using the same steps as described in detail above.

In this section, we present experimental data obtained from two sets of experiments, corresponding to the same bead at the same height in the actin network but at different times. First a set (a) of both passive and active measurements was recorded. Then position calibration with $500 \mathrm{~nm}$ oscillations at $2 \mathrm{~Hz}$ was performed. These measurements allowed a determination of $\beta^{\text {(SIN) }}$ in the actin solution. Thereafter, the second set of measurements (b) again including both passive and active measurements was recorded. Presumably, the 


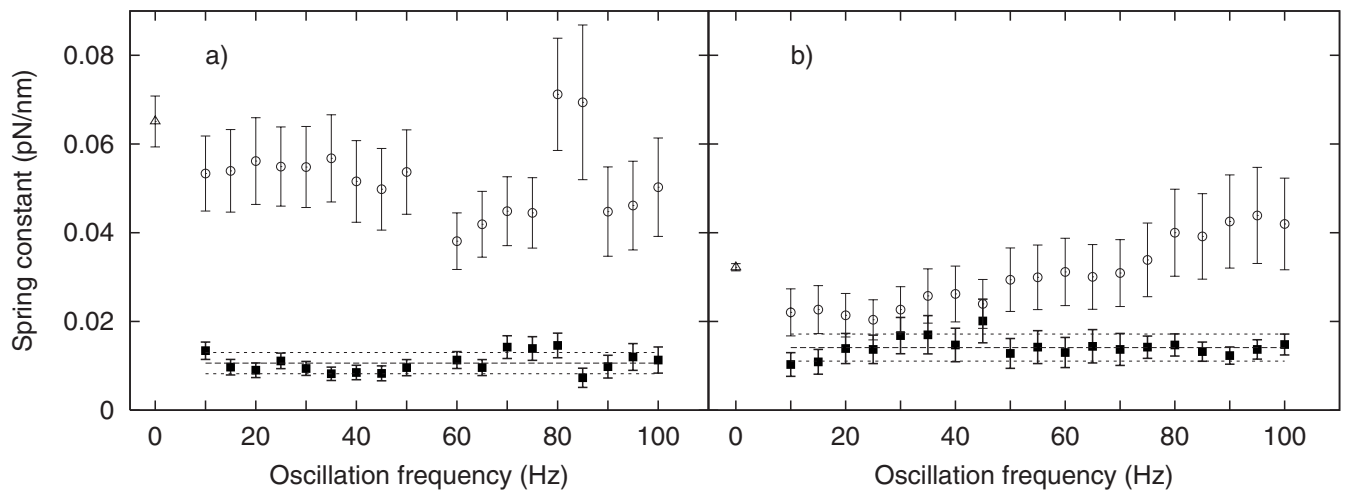

FIG. 6. Filled squares with error bars: Estimates for $\kappa$ as obtained at the particular frequency. Long-dashed line gives $\kappa^{\text {(av) }}$ with dotted lines indicating \pm two times the standard deviation. Open circles with error bars: $1 /\left|\chi\left(f_{k}\right)\right|$, symbolizing the effective spring constant felt by the bead. Open triangles with error bars: $k_{B} T /\left\langle x^{2}\right\rangle$, also symbolizing the effective spring constant felt by the bead. Parts (a) and (b) are measured for the same bead but between the two measurements, the actin network was disturbed and probably partially disrupted by large amplitude oscillations of the stage. It illustrates that we find the same trap properties, even when the effective spring constant felt by the bead is different.

large amplitude oscillations of the stage for the positional calibration resulted in a partial disruption of the actin network, as also indicated by microscope observations that the embedded beads show different behavior in the two cases (a) and (b).

In Fig. 6, the results of the trap calibration are displayed. The resulting average spring constant of the optical trap, plotted as long-dashed lines with short-dashed lines indicating two times the standard deviation, equals $\kappa=(1.06 \pm 0.12) \cdot 10^{-2} \mathrm{pN} / \mathrm{nm}$ in the experiments in part (a) of the figure and $\kappa=(1.41 \pm 0.15) \cdot 10^{-2} \mathrm{pN} / \mathrm{nm}$ in the experiments in part (b) of the figure. Similar results have been obtained in other experiments with similar conditions.

For comparison, in an experiment in water with the same laser settings, we find for a bead of the same size trapped at the same depth, a spring constant of order $\kappa_{\text {water }}=(1.4-1.8) \cdot 10^{-2} \mathrm{pN} / \mathrm{nm}$. Correction for the refractive index difference between a water solution and the actin solution is difficult as we do not know the refractive index of the latter, yet an analysis as in Ref. 22 [Eq. (3)], with an estimate of $n_{\text {actin }}$ between that of water $(n=1.33)$ and that of a cell $(n \sim 1.5)$, suggests that the spring constant of the trap in the actin solution could be as low as $34 \%$ of that in water. This analysis is based on a continuous medium model. Ide- ally, our solution of actin is a homogeneous network, and our analysis relies on that it is so, yet inhomogeneities might occur. With that in mind, our experimental results, $\kappa_{\text {actin }} / \kappa_{\text {water }} \sim 0.8$, appear reasonable.

We speculate that improvements to our description and the active-passive calibration method would require a description of the actin solution as a two-component medium, in which the bead is mainly situated in water, trapped in a water-filled cage between the actin filament.

Figure 6 also illustrates other strengths and weaknesses of methods estimating trap stiffnesses in viscoelastic media: As mentioned above, the two sets of measurements in (a) and (b) differ in time and history, thus the properties of the actin network are likely to be different in the two cases but the properties of the trap are expected not to differ. Within two standard deviations, we find the same trap strength in (a) and (b), whereas the total spring constant felt by the bead is clearly different in the two cases. In the work of Atakhorrami et al., 5 the suggested approximative method to find the trap stiffness in situ is based on the limiting behavior of the total spring constant felt by the bead as $f \rightarrow 0$. Our results illustrate a possible shortcoming of that method, as already alluded to by these authors: Our case (a) corresponds to a medium that is highly elastic in which case the total spring

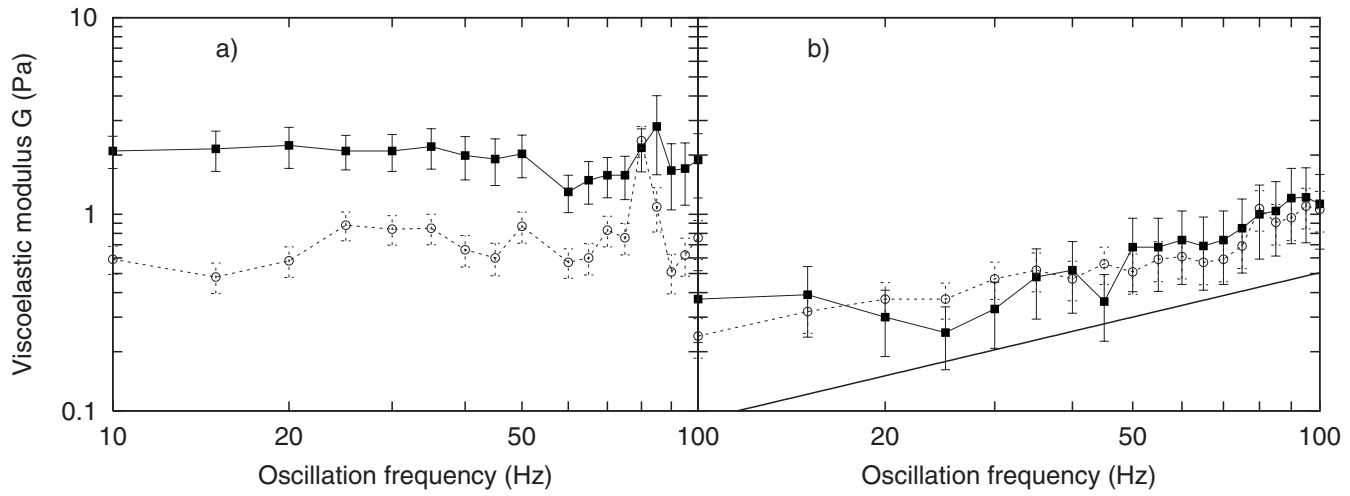

FIG. 7. Viscoelastic moduli. Filled squares with solid line give $G^{\prime}$ whereas open circles with dotted line give $G^{\prime \prime}$. Parts (a) and (b) show the data obtained from the same bead in an actin solution, but in between the two measurements, the actin solution was partially disrupted by oscillations of the stage of an amplitude of $500 \mathrm{~nm}$ at $2 \mathrm{~Hz}$. In part (a) of the figure, the solution of filamentous actin displays the characteristics of a purely elastic network whereas in part (b), the results demonstrate viscoelastic characteristics. To guide the eye, the thick solid line is proportional to $f^{0.75}$. 
constant felt by the probe is clearly different from the spring constant of the trap. On the contrary, our case (b) shows an example where we expect our evaluation of the trap stiffness to agree with an estimate using the procedure suggested by Atakhorrami et al. ${ }^{5}$

As a final comparison, the figure also shows the result of the most direct analysis of the data, based on the equipartition theorem and a direct calculation of $\left\langle x^{2}\right\rangle$ from the raw time series. According to the equipartition theorem, $\left\langle x^{2}\right\rangle=k_{B} T\langle\chi(0)\rangle$, or, in the limit of $\omega \rightarrow 0, k_{B} T /\left\langle x^{2}\right\rangle=\kappa$ $+\omega \operatorname{Im}(\tilde{\gamma}(\omega))$, a result including both elastic components from the optical trap and from the actin filament network. It may thus be compared to $1 /|\chi(f)|$ as $f \rightarrow 0$.

Figure 7 displays the viscoelastic moduli of the actin network in the same two cases as in Fig. 6. The moduli are determined under the assumption that the network is in equilibrium and the error bars reflect the corresponding statistical errors. The moduli may directly be compared to other measurements of viscoelastic properties, in in vitro actin solutions. Literature states that, in a limited frequency range, $G^{\prime}$, $G^{\prime \prime} \propto f^{0.75}, 23$ whereas for low frequencies, elasticity dominates, resulting in a plateau in $G^{\prime}, G^{\prime \prime}$. Both features are also displayed in our data, and we find reasonable agreement with previously published values for the moduli ${ }^{24}$ at similar actin concentration. To guide the eye, the lower thick solid line in part (b) of the figure is proportional to $f^{0.75}$.

\section{CONCLUSION}

In this paper, we present a way to calibrate single beam optical tweezers in situ in viscoelastic media, through a combination of passive and active recordings on the same system. We stated calibration equations for the special case of sinusoidal driving in terms of the amplitudes $A_{P}, A_{S / L}$, and the phase difference $\Delta \phi$ and we tested the method in water and entangled F-actin. The so-called FDT method is based on linear response theory in continuous media. No specific model for the friction felt by the trapped particle in the viscoelastic medium is assumed. Therefore, no prior knowledge of viscoelastic parameters of the medium or the shape of the particle is required. It is required however, that all particle motion takes place within the harmonic region of the trapping potential. Furthermore, for a linear response, the active part of the experiment must only inflict small perturbations to the trapped particle. In particular, we foresee that the FDT method will be applicable in investigations of how forces are generated in a biological cell. An important concern when applying the FDT method to living cells is the presence of bioactive processes which renders the FDT invalid in certain frequency ranges: In an actin network with molecular motors, data for fluctuating beads show disagreement with the fluctuation-dissipation theorem at frequencies below $10 \mathrm{~Hz} .^{25}$ However, modern piezostages can be moved with frequencies up to several hundred $\mathrm{Hz}$ and acousto-optic deflector can deflect lasers even with frequencies of several kHz. At such high frequencies, no significant nonequilib- rium contributions to the power spectrum are expected and the FDT would remain valid. It remains to be seen if frequency ranges of bioactive processes will reveal themselves in experimental data sufficiently and clearly such that the experimenter will know that data at these frequencies are to be excluded from the process of determination of the trap characteristics.

\section{ACKNOWLEDGMENTS}

This work was supported by the European Commission through the Marie Curie-Early Stage Training on Biomimetic Systems (Contract No. MEST-CT-2004-504465) and by the European Union FP6 through the Network of Excellence BioSim (Contract No. LSHB-CT-2004-005137) and by the Danish research councils. We thank Thomas Heimburg, Martha Alvarez, Susan Roelofs, and Christoph F. Schmidt for inspiring discussions.

${ }^{1}$ W. J. Greenleaf, M. T. Woodside, and S. M. Block, Annu. Rev. Biophys. Biomol. Struct. 36, 171 (2007).

${ }^{2}$ K. Berg-Sørensen and H. Flyvbjerg, Rev. Sci. Instrum. 75, 594 (2004).

${ }^{3}$ S. F. Tolić-Nørrelykke, E. Schaffer, J. Howard, F. S. Pavone, F. Julicher, and H. Flyvbjerg, Rev. Sci. Instrum. 77, 103101 (2006).

${ }^{4}$ E.-L. Florin, A. Pralle, E. H. K. Stelzer, and J. K. H. Hörber, Appl. Phys. A: Mater. Sci. Process. 66, S75 (1998).

${ }^{5}$ M. Atakhorrami, J. I. Sulkowska, K. M. Addas, G. H. Koenderink, J. X. Tang, A. J. Levine, F. C. MacKintosh, and C. F. Schmidt, Phys. Rev. E 73, 061501 (2006).

${ }^{6}$ D. Mizuno, D. A. Head, F. C. MacKintosh, and C. F. Schmidt, Macromolecules 41, 7194 (2008).

${ }^{7}$ A. C. Richardson, S. N. S. Reihani, and L. B. Oddershede, Opt. Express 16, 15709 (2008).

${ }^{8}$ K. C. Neuman and S. M. Block, Rev. Sci. Instrum. 75, 2787 (2004).

${ }^{9}$ K. C. Vermeulen, J. van Mameren, G. J. M. Stienen, E. J. G. Peterman, G. J. L. Wuite, and C. F. Schmidt, Rev. Sci. Instrum. 77, 013704 (2006).

${ }^{10}$ B. Schnurr, F. Gittes, F. C. MacKintosh, and C. F. Schmidt, Macromolecules 30, 7781 (1997).

${ }^{11}$ F. Gittes, B. Schnurr, P. D. Olmsted, F. C. MacKintosh, and C. F. Schmidt, Phys. Rev. Lett. 79, 3286 (1997).

${ }^{12}$ I. M. Tolić-Nørrelykke, E. L. Munteanu, G. Thon, L. B. Oddershede, and K. Berg-Sørensen, Phys. Rev. Lett. 93, 078102 (2004).

${ }^{13}$ M. Fischer and K. Berg-Sørensen, J. Opt. A, Pure Appl. Opt. 9, S239 (2007).

${ }^{14}$ M. Fischer, Ph.D. thesis, Copenhagen University, Niels Bohr Institute, Faculty of Science, 2007.

${ }^{15}$ R. Kubo, M. Toda, and N. Hashitsume, Statistical Physics (Springer, Heidelberg, 1985), Vol. 2.

${ }^{16}$ L. Oddershede, S. Grego, S. F. Nørrelykke, and K. Berg-Sørensen, Probe Microsc. 2, 129 (2001).

${ }^{17}$ J. A. Spudich and S. Watt, J. Biol. Chem. 246, 4866 (1971).

${ }^{18}$ A. D. Mehta, J. T. Finer, and J. A. Spudich, Methods Cell Biol. 55, 47 (1997).

${ }^{19}$ A. Rohrbach, H. Kress, and E. H. K. Stelzer, Opt. Lett. 28, 411 (2003).

${ }^{20}$ J. H. G. Huisstede, K. O. van der Werf, M. L. Bennink, and V. Subramaniam, Opt. Express 13, 1113 (2005).

${ }^{21}$ E. Peterman, F. Gittes, and C. Schmidt, Biophys. J. 84, 1308 (2003).

${ }^{22}$ R. R. Brau, J. M. Ferrer, H. Lee, C. E. Castro, B. K. Tam, P. B. Tarsa, P. Matsudaira, M. C. Boyce, R. D. Kamm, and M. J. Lang, J. Opt. A, Pure Appl. Opt. 9, S103 (2007).

${ }^{23}$ F. Gittes and F. C. MacKintosh, Phys. Rev. E 58, R1241 (1998).

${ }^{24}$ T. G. Mason, T. Gisler, K. Kroy, E. Frey, and D. A. Weitz, J. Rheol. 44, 917 (2000).

${ }^{25}$ D. Mizuno, C. Tardin, C. F. Schmidt, and F. C. MacKintosh, Science 315, 370 (2007). 